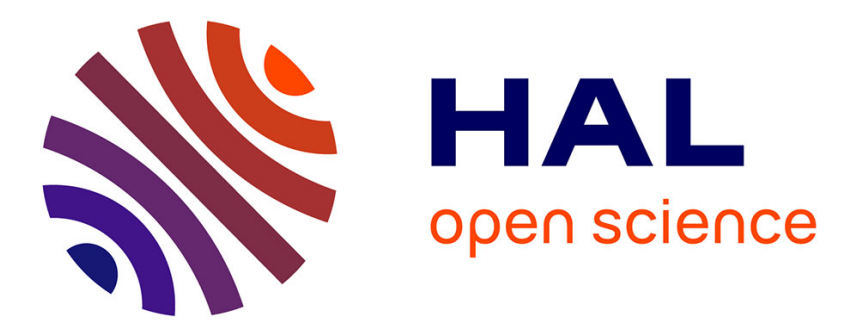

\title{
Une approche de l'expérience et de l'identité parentales pour l'étude des spécificités des discours des hommes et des discours des femmes
}

\author{
Christine Bouissou, Geneviève Bergonnier-Dupuy
}

\section{- To cite this version: \\ Christine Bouissou, Geneviève Bergonnier-Dupuy. Une approche de l'expérience et de l'identité parentales pour l'étude des spécificités des discours des hommes et des discours des femmes. Connex- ions, 2004, 2 (82), pp.186-201. 10.3917/cnx.082.0185 . halshs-01254485}

\section{HAL Id: halshs-01254485 \\ https://shs.hal.science/halshs-01254485}

Submitted on 12 Jan 2016

HAL is a multi-disciplinary open access archive for the deposit and dissemination of scientific research documents, whether they are published or not. The documents may come from teaching and research institutions in France or abroad, or from public or private research centers.
L'archive ouverte pluridisciplinaire HAL, est destinée au dépôt et à la diffusion de documents scientifiques de niveau recherche, publiés ou non, émanant des établissements d'enseignement et de recherche français ou étrangers, des laboratoires publics ou privés. 
Revue Connexions, $n^{\circ} 82,2004$, pp.185-201

\title{
Une approche de l'expérience et de l'identité parentales par l'étude des spécificités des discours des hommes et des discours des femmes
}

\author{
BOUISSOU Christine * \\ BERGONNIER-DUPUY Geneviève** \\ * Maître de conférences en psychologie
}

${ }^{* *}$ Maître de conférences en sciences de l'éducation

EA n¹589, Centre de Recherche en Éducation et Formation, Équipe Éducation Familiale et Interventions Sociales auprès des Familles, Université Paris X Nanterre

\section{Résumé}

Dans cette recherche nous nous sommes intéressées à l'expérience de la parentalité de vingt-cinq couples hétérosexuels, professionnellement actifs et parents d'un jeune enfant. Chaque parent nous a accordé un entretien, analysé quantitativement et qualitativement. Nous avons ainsi pu mettre en évidence l'importance des rôles sociaux de sexe et de la dynamique intersubjective : l'exercice de la parentalité en couple est une expérience individuelle et partagée qui se définit au cours des interactions quotidiennes, et chacun participe de l'identité du partenaire et de la permanence des rôles sociaux de sexe.

\section{Contexte théorique}

\section{La parentalité, une expérience sociale et identitaire}

Les évolutions sociales et la complexification croissante des contextes de vie qu'analysent les sociologues (Corcuff, 2003, Dubet, 1994, Boltanski \& al., 1991, Élias, 1973), ont des conséquences importantes sur les processus de socialisation. Le monde social actuel n'est plus réductible à un système intégré et ordonné selon des règles unifiées. L'homme est «pluriel », connaît des processus de socialisation multiples, construit des dispositions variées, parfois en concurrence (Lahire, 2002). Ses conduites combinent des rationalités et des logiques différentes et le rôle de la subjectivité s'en trouve redoublé. Selon Dubet (1994), le seul principe d'unité de l'expérience sociale est le travail sur lui-même que poursuit chacun d'entre nous, afin de se percevoir comme l'auteur de sa propre vie. La notion d' « expérience » émerge des travaux sur la 
Revue Connexions, $\mathrm{n}^{\circ} 82,2004$, pp.185-201

socialisation : «si le principe d'unité de l'ordre social ne réside ni dans la structure fonctionnelle des rôles, ni dans les valeurs transcendantes qui s'imposent à tous, il doit être recherché dans le travail des individus et des divers acteurs pour construire cet ordre, ce que l'on peut appeler leur expérience » (Dubet, 2000, p 75). Ce travail psychique, identitaire, subjectif, donne à la vie adulte un caractère d' « immaturité » (Boutinet, 1998) et nécessite des compétences de réflexivité ; on entend par là, l'aptitude d'acteurs constamment engagés dans le flot des conduites quotidiennes à comprendre ce qu'ils font pendant qu'ils le font (Giddens, 1987). Le renforcement de la réflexivité à l'époque contemporaine doit se comprendre comme la conséquence du relâchement des contraintes, de l'assouplissement des rôles sociaux et des normes de conduite. La désinstitutionnalisation de la famille, la flexibilité et l'instabilité des emplois, ainsi que la multiplicité des sollicitations de la vie moderne exigent de l'individu une autoanalyse et une redéfinition permanente de ses choix. Les rôles et normes sociaux n'étant plus clairement établis, il importe de s'interroger davantage sur la façon de se comporter. Le répertoire comportemental reste ouvert, entraînant une importante activité de réflexion dans et sur l'action (Schön, 1994). Les contextes sociaux (ou «modèles régionaux d'action », Boltanski \& al., 1991) sont divers, véhiculent des normes différentes, parmi lesquelles évolue l'individu, qui doit apprendre à s'y adapter et à jouer avec elles ; les compétences et dispositions auxquelles il fait appel varient selon les situations, l'inhibition des unes étant nécessaire à l'expression et au développement des autres. Sur le plan psychique, elles entrent parfois en contradiction (Lahire, 2002). La sociologie contemporaine a pris une orientation psychologique et cognitive : l'individu raisonne, analyse, réfléchit, il n'est pas un automate social englué dans les croyances et les normes. Son activité psychique est appréhendée en termes de pluralité, de «travail » et d'articulation identitaires. Vivant des expériences de socialisation diverses et hétérogènes, l'individu ne se construit pas sans contradictions psychologiques (Lahire, 2001).

Dans cette perspective, la parentalité est une expérience sociale et individuelle attachée à un processus de socialisation secondaire (Berger \& al., 1986), que la personne doit chercher à articuler avec ses autres expériences. Les parents ont à gérer et à combiner des logiques différentes afin de forger la continuité et la stabilité de leur expérience ; il s'agit d'un travail 
Revue Connexions, $n^{\circ} 82,2004$, pp.185-201

identitaire spécifique grâce auquel ils s'efforcent de reconstituer une unité de leur expérience, car cohérence interne et la continuité dans le temps sont deux dimensions essentielles de l'identité (Tap, 1988). Ainsi, en raison de leurs multiples insertions sociales et occupations, et afin de maintenir le sentiment de stabilité et de cohérence interne, d'articuler les différents domaines de vie, les hommes et les femmes ont à mener un travail d'unification psychique. C'est le cas des femmes, dont la vie est tiraillée entre plusieurs modèles «femme active », «mère de famille », « célibataire », «conjointe » (Cicchelli, 2001). C'est le cas des hommes aussi, car l'évolution de l'identité sociale masculine les incite à redéfinir leurs conduites.

\section{La parentalité chez les femmes et les hommes}

On considère que la parentalité se décline et se traduit de manière spécifique pour les uns et les autres. D’une part, parce que le lien entre parentalité et activité professionnelle ne se joue pas de la même manière pour les hommes et les femmes ; en effet, de l'avis d'un certain nombre d'analystes, le mariage et la maternité constituent un vrai handicap pour la carrière des femmes ; à tel point que « quand un homme se marie, sa carrière fait un bond en avant, celle de la femme stagne ou recule » (Bulard, 2003). D'autre part, le rapport au temps, linéaire et continu pour les hommes, plus fragmenté pour les femmes, est une dimension essentielle de la différenciation entre les sexes (Boutinet, 1998). « Les hommes qui ne sont pourtant pas, il s'en faut de beaucoup, affranchis des questions du temps, des cycles, de la fatigue, de la maladie, du vieillissement et de la mort, ont tendance à se défausser d'une bonne part des contraintes relatives à la temporalité, sur les femmes auxquelles ils délèguent les questions du temps, de la variabilité, de la disponibilité, etc. délégation qui est, en fait, plutôt une façon de se débarrasser : socialement et matériellement bien sûr, mais mentalement aussi, car ce faisant ils occultent leurs angoisses par rapport aux rythmes biologiques, aux rythmes domestiques, aux rythmes des « mouvements de vie et de mort » (Dejours, 1988). Par ailleurs, comme l'a montré de Singly (1990), la spécialisation « homme au travail », « femme au foyer » est fonctionnelle dans un contexte culturel individualiste, dominé par deux tensions majeures contradictoires : la raison humanitaire (attachée aux valeurs humanistes telles que l'égalité entre tous) et la raison utilitaire (attachée au principe de réussite individuelle). Ainsi, la spécialisation sexuelle au sein du couple permet à l'homme de garantir la 
Revue Connexions, $n^{\circ} 82,2004$, pp.185-201

fortune individuelle et à la femme, la qualité humaine ; chacun représente au mieux une des deux exigences du monde social. La dualité conjugale favorise l'équilibre du couple dans la mesure où elle résout certaines contradictions et « dissout magiquement les contradictions de l'homme dual » (ibid., p.150). Ces deux pôles « raison humanitaire / raison utilitaire » correspondent à deux formes différentes de réalisation de soi et de «production d'œuvres » qui participent à la consolidation de l'identité (Meyerson, 1948, Molinier, 2002).

\section{La parentalité en couple}

Si être parent est une expérience individuelle, elle est aussi, la plupart du temps, partagée avec un conjoint, co-définie, dans une dynamique interactionnelle et intersubjective, où chacun participe de l'identité de l'autre significatif. Nous devenons nous-mêmes à travers un échange incessant avec ceux que nous considérons comme des « autres significatifs » (Mead, 1933). « Dans la banalité de l'intersubjectivité de tous les jours, nous forgeons nos repères et renforçons l'illusion de leur indubitable légitimité, nous composons l'ethos (individuel et micro-collectif) qui détermine nos actions et leur donne sens »(Kaufmann, 1988, p.11). Si la parentalité constitue une étape de socialisation essentielle, l'entrée en couple est un autre temps fort d'évolution et de construction identitaire. Kaufmann a montré qu'elle correspond à une rupture profonde dans l'ordre des significations, acte «dramatique » par lequel deux étrangers se rencontrent et se redéfinissent (Kaufmann, 1994). II s'agit d'un processus «nomique » particulièrement puissant, un « engagement social qui crée pour l'individu une sorte d'ordre dans lequel sa vie prend un sens » (Berger \& al., 1988, p.6). Et ce, même si l'entrée en couple se fait aujourd'hui plus progressivement qu'autrefois, et ne semble pas entraîner de bouleversements radicaux du mode de vie. C'est pourtant « dans l'interaction quotidienne, dans les rapports interpersonnels que les partenaires se redéfinissent mutuellement, surtout au début » (Kaufmann, 1994, p. 319). A l'espace de socialisation spécifique à la jeunesse, privilégiant les rapports de «personne à personne ", succède un second temps, celui de la structuration de l'organisation ménagère ; après la personnalité de jeunesse, s'installe une personnalité de l'âge domestique. A chaque reformulation identitaire, le couple avance d'un degré dans l'intégration ménagère et structure toujours davantage son organisation domestique. On note chez les couples d'aujourd'hui, le refus 
Revue Connexions, $n^{\circ} 82,2004$, pp.185-201

des rôles déjà construits, la volonté d'improviser, d'inventer les rôles domestiques, de choisir « qui fera quoi », sans idées a priori. Toutefois « geste après geste des habitudes et des règles d'interaction se sédimentent, des conceptions s'imposent, des spécialités se constituent. Au terme de cette accumulation, les rôles peu à peu se dessinent » (Kaufmann, 1991). Il y a à la fois refus et reconstruction des rôles par les membres du couple ; la dynamique intersubjective participe de la pérennité des rôles sociaux de sexe. La négociation des habitudes au sein du couple suit un mouvement progressif, véritable « travail en sourdine » (Kaufmann, 1994).

Ces réflexions légitiment l'analyse des comportements parentaux en termes de rôles et la tentative d'articuler déterminismes sociaux et créativité individuelle, prise de rôle et expérience singulière, en montrant que les individus s'ajustent, tâtonnent, et sont redéfinis par une réalité qu'ils ont contribué à redéfinir. Au sein du couple, la réalité est objectivée en commun et se stabilise. L'engagement dans des rôles et la négociation d'ajustements dans l'interaction sont peu visibles et peu conscients. Une idée force qui sous-tend les réflexions développées dans cet article est que l'activité de perception, d'interprétation, de mise en signification du monde est au cœur du processus de socialisation : le réel (et le sens donné au réel) n'existe pas en tant que tel, il est construit, même s'il finit par apparaître à l'individu sous un aspect d'objectivité (Berger \& al., 1986). D’où notre intérêt pour l'étude de la construction « en sourdine » de rôles « qui ne se disent pas ». Dans cette perspective constructiviste, le choix méthodologique d'une démarche compréhensive procédant par entretien est pertinent. La parentalité en tant qu'expérience sociale conduit l'individu à construire du sens, à s'approprier des symboles socialement et culturellement élaborés. La production de parole est l'occasion d'une externalisation de cette activité, et en cela elle est une pratique sociale (Reinert, 1997).

\section{Méthodologie de la recherche}

L'étude que nous avons menée répondait à un appel d'offre de la Caisse Nationale des Allocations Familiales (Bergonnier-Dupuy \& Bouissou 2003 ; Bouissou \& Bergonnier-Dupuy, 2004). On a analysé l'organisation familiale à l'arrivée de l'enfant, la négociation des arrangements au sein du 
Revue Connexions, $n^{\circ} 82,2004$, pp.185-201

couple - en termes de partage des tâches et d'articulation entre sphère domestique et autres domaines de vie - en cherchant à comprendre ce qui distingue les deux sexes. Nous avons souhaité explorer de multiples dimensions de l'expérience et de l'identité parentale. Ont ainsi été analysées, aussi bien les actions et les pratiques des parents, que leurs idées, représentations au sujet de leurs actions et de l'enfant, ou encore le vécu de leur expérience et l'évaluation qu'ils en font. Par ailleurs, il a semblé important de resituer les personnes dans leur contexte de vie ; le questionnement a donc porté sur les relations avec les partenaires : conjoint, enfant, parents, amis, collègues, professionnels s'occupant de l'enfant et les autrui «abstraits » (Mead, 1933, Wallon, 1946). Par ailleurs, l'expérience parentale a été abordée de manière descriptive (la narration de l'expérience est essentielle, Ricoeur, 1990), mais aussi d'un point de vue autoévaluatif et normatif, au regard des idéaux de la personne.

Le temps est également une dimension importante quand il s'agit d'identité, le sentiment de continuité de soi à travers les changements, étant une «charpente » essentielle de l'identité (Tap, 1988). La parentalité est un processus qui concerne une périodicité longue, et est bien sûr susceptible d'évolutions.

C'est à partir de ces jalons que nous avons construit une grille d'entretien. L'entretien individuel et semi-directif nous a semblé un choix judicieux pour l'étude de l'identité ; il permet d'appréhender la manière dont la personne fait « une synthèse de l'hétérogène », un « travail discursif de mise en cohérence du moi malgré la multiplicité des épisodes d'une vie (Corcuff, 2003, p.79).

\section{Grille d'entretien}

\begin{tabular}{|c|c|}
\hline LES DOMAINES EXPLORÉS PAR L'ÉTUDE & LES QUESTIONS \\
\hline $\begin{array}{l}\text { Organisation concrète au sein du couple parental } \\
\text { On cherche à comprendre comment s'organise la vie } \\
\text { quotidienne et les relations familiales depuis la } \\
\text { naissance de l'enfant }\end{array}$ & $\begin{array}{l}\text { Qui fait quoi auprès de l'enfant ? Quel mode de } \\
\text { garde? Comment la vie s'organise-t-elle entre les } \\
\text { deux parents : partage des tâches de soin et } \\
\text { d'éducation, partage des autres tâches quotidiennes } \\
\text { (semaine et week-end) Comment cela s'est mis en } \\
\text { place? (ajustements, relations à l'intérieur du couple) }\end{array}$ \\
\hline $\begin{array}{l}\text { Articulation avec les autres domaines de vie } \\
\text { On aborde la question des insertions extra-familiales, } \\
\text { professionnelles et autres de la personne }\end{array}$ & $\begin{array}{l}\text { Comment la personne concilie-t-elle ses différentes } \\
\text { occupations ? Quel est son réseau social et de quel } \\
\text { soutien social bénéficie-t-elle? Quels sont ses } \\
\text { rapports avec les autres partenaires éducatifs (famille, } \\
\text { professionnels, amis)? }\end{array}$ \\
\hline $\begin{array}{l}\text { Représentations de l'éducation et de l'enfant } \\
\text { L'expérience de la parentalité concerne également les } \\
\text { représentations concernant l'enfant (ses besoins, les }\end{array}$ & $\begin{array}{l}\text { Quelle idée de l'éducation la personne se fait-elle, } \\
\text { quels sont les besoins de l'enfant? Que veut-elle } \\
\text { transmettre? Quelle idée a-t-elle de l'avenir, quels }\end{array}$ \\
\hline
\end{tabular}


Revue Connexions, n॰82, 2004, pp.185-201

\begin{tabular}{|c|c|}
\hline valeurs qu'on veut lui transmettre) & sont ses projets vis-à-vis de l'enfant? \\
\hline $\begin{array}{l}\text { Estime de soi et Sentiment de compétence } \\
\text { On cherche à cerner l'image de soi du parent, les } \\
\text { compétences qu'ils s'attribue, la relation entre } \\
\text { appréciation personnelle et comparaison sociale }\end{array}$ & $\begin{array}{l}\text { La personne dirait-elle qu'elle se sent compétente, et } \\
\text { si oui en quoi particulièrement ? Comment se } \\
\text { positionne-t-elle par rapport aux autres (concrets : les } \\
\text { partenaires et abstraits : les parents en général) ? Et } \\
\text { comment se perçoit-elle par rapport à son conjoint et } \\
\text { par rapport à l'image idéale du "bon " parent, du } \\
\text { parent compétent? Comment se voit-elle par rapport } \\
\text { à ses propres parents (ou autres figures parentales)? }\end{array}$ \\
\hline $\begin{array}{l}\text { L'expérience de la réalité } \\
\text { On cherche à aborder la perception que le sujet a de } \\
\text { lui-même dans la réalité quotidienne, ainsi que } \\
\text { l'image idéale de soi et la manière dont il gère } \\
\text { l“ écart " entre ces deux aspects }\end{array}$ & $\begin{array}{l}\text { Quelles sont les contraintes que la personne } \\
\text { rencontre, qu'est-ce qui la “limite ", l'empêche de faire } \\
\text { ce qu'elle aurait souhaité, ou au contraire, la conduit à } \\
\text { faire ce qu'elle ne souhaitait pas? }\end{array}$ \\
\hline $\begin{array}{l}\text { Les occasions d'une analyse réflexive } \\
\text { On souhaite cerner le «travail » d'articulation, } \\
\text { d'unification identitaire, de réflexion sur l'expérience } \\
\text { parentale }\end{array}$ & $\begin{array}{l}\text { La personne a-t-elle des occasions d'évoquer son } \\
\text { vécu en tant que parent, avec d'autres? Si oui, avec } \\
\text { qui et sous quelle forme? A quelles occasions, pour } \\
\text { quels bénéfices? Est-ce qu'être parent ou devenir } \\
\text { parent est quelque chose qui « s'apprend »? A-t-elle, } \\
\text { en tant que parent, des besoins, satisfaits ou non? } \\
\text { lesquels? }\end{array}$ \\
\hline
\end{tabular}

L'ordre du questionnement a été établi de sorte à solliciter progressivement au fil de l'entretien, une plus forte implication (tant au plan cognitif qu'émotionnel) ; toutefois, le choix de la méthode semi-directive suppose de suivre un guide de questionnement souple : il indique les domaines à explorer, tout en laissant l'interviewer libre de s'adapter à la parole de l'interviewé, d'approfondir certains points qui lui semblent susceptibles d'enrichir l'analyse, de faire évoluer le questionnement.

\section{La population de l'étude}

L'échantillon est constitué de vingt-cinq couples hétérosexuels, parents de jeunes enfants (de un à trois ans), citadins, insérés professionnellement tous les deux, rencontrés par l'intermédiaire de crèches et de haltes-garderies. Un certain nombre d'informations ont été recueillies : Age des parents, Activité et Implication professionnelle, Niveau d'études, Situation conjugale, Nombre et âge des enfants du couple, Nombre de frères et sœurs du parent, Profession des grands-parents.

Pour situer socioculturellement et économiquement les personnes, nous avons construit une variable multidimensionnelle, définie par la profession et le niveau d'études de chaque parent, la trajectoire sociale familiale (à partir de la situation des grands-parents), et le lieu d'habitation. Elle se décline en 3 modalités : 
Revue Connexions, $n^{\circ} 82,2004$, pp.185-201

- Classe moyenne-populaire: profession d'employé/artisan; parents du couple issus du prolétariat

- Classe moyenne-supérieure : trajectoire sociale familiale ascendante, origines socioculturelles sensiblement différentes entre les deux membres du couple

- Classe bourgeoise: niveaux économique et culturel élevés, origines socioculturelles homogènes entre les deux conjoints.

Les critères choisis pour définir les catégories n’ont pas été décidés « a priori » mais sont ceux qui permettent de discriminer au mieux la population d'étude. Les profils sociologiques des parents sont relativement divers; cependant la procédure d'échantillonnage n’a pu éviter une surreprésentation des classes sociales favorisées, et une sous-représentation des milieux les plus modestes. Les critères d'échantillonnage (notamment la nécessité de retenir des couples insérés professionnellement) ont écarté de l'étude les familles en situation de grande précarité. A cela s'est rajoutée la difficulté à engager dans l'étude, les personnes issues de milieux socioculturels défavorisés.

\section{Méthode d'analyse des discours}

1 - la lecture minutieuse des entretiens a permis une analyse qualitative de leur contenu et la compréhension de leur logique propre.

2 - une analyse quantitative de données textuelles informatisée (logiciel Alceste) permet de dégager les structures signifiantes les plus fortes d'un corpus, - par le calcul des fréquences d'apparition de mots et de séquences de mots - et de mettre en évidences des «mondes lexicaux » (Reinert, 1997). Le logiciel procède à des tris croisés, des analyses factorielles des correspondances (AFC) et des classifications hiérarchiques descendantes (CHD). Certaines analyses ont porté sur l'ensemble des discours, d'autres se sont attachées à mettre en évidence les particularités des discours des hommes et des discours des femmes. 


\section{Résultats}

\section{Logistique domestique et activité professionnelle}

II apparaît que l'expérience de la parentalité prend des formes différentes pour les hommes et pour les femmes. D'une part, la « logistique domestique » revient largement aux femmes et d'autre part, elles ont à gérer - plus que les hommes-, l'articulation entre la sphère domestique et la sphère professionnelle. L'exercice de la parentalité est en effet attaché à des activités spécifiques pour les uns et les autres ; les champs éducatifs sont répartis en fonction du sexe des parents : la logistique, l'organisation de la vie domestique et un grand nombre de tâches ménagères sont largement prises en charge par les mères. Les pères excellent (de leur propre avis et de l'avis de leur conjointe) dans les activités ludiques avec l'enfant. Ce résultat vient corroborer des observations nombreuses (Bihr \& al., 2002, Cicchelli, 2001).

Sur le plan de l'articulation vie familiale - vie professionnelle, l'équilibre n'est pas toujours garanti, notamment pour les femmes. Pour elles, le travail est ce qui prive de l'enfant ; elles sont à la recherche de compromis, de conciliation, tiraillées souvent entre activité professionnelle et disponibilité familiale, car tout en maintenant une insertion professionnelle, la plupart d'entre elles a été conduite à des aménagements (par exemple en choisissant de travailler à temps partiel, ou en organisant leur activité pour pouvoir travailler à leur domicile). Les femmes cherchent à articuler les deux domaines de vie que sont la sphère professionnelle et la sphère domestique, manifestant par là un « double attachement au rôle maternel et au statut de travailleuse " (Junter-Loiseau, 1997, p. 85).

L'exercice de la parentalité est décrit comme un ensemble de plus ou moins " petits " problèmes à régler, dans l'urgence. Ce n'est pas exactement le cas des hommes. Ils se reconnaissent plus investis dans leur activité professionnelle, moins dans l'organisation domestique ; l'arrivée de l'enfant n'a pas eu de répercussion notable sur leur activité professionnelle. La vie familiale leur apporte réconfort, et contraste avec le monde professionnel.

Cette « répartition » semble s'effectuer sans heurts. Sans doute parce qu'il s'agit d'un processus interactif : l'investissement professionnel des hommes renforce - et est renforcé par - la prise en charge par les femmes des responsabilités domestiques. Les uns sont incités ainsi à devenir les 
Revue Connexions, $\mathrm{n}^{\circ} 82,2004$, pp.185-201

principaux pourvoyeurs de ressources économiques et symboliques de la famille, les autres à s'engager dans la sphère domestique (Bihr \& al., 2002).

Décrire ce processus en termes d'interaction c'est insister sur le fait que les attitudes des uns et des autres se renforcent mutuellement (Bergonnier-Dupuy, 2000).

\section{Les référents identitaires}

Le processus d'élaboration identitaire se joue différemment pour les femmes et les hommes, sans doute en raison de l'importance des rôles de sexes et des figures identificatoires qui interviennent dans cette construction. Au cours des entretiens, alors qu'une question incitait les parents à se définir par rapport à leur conjoint, ils ont dans l'ensemble peu répondu : hommes et femmes ne se sont pas comparés entre eux. En revanche chacun a largement évoqué ses propres parents et particulièrement son parent de même sexe. Les parents interviewés s'affirment à la fois différents de celui-ci (en particulier les hommes, qui se perçoivent plus proches de leur enfant que leur propre père ne l'a été avec eux), et s'en inspirent pour définir les principes éducatifs : l'éducation reçue semble être la référence à partir de laquelle ils définissent celle qu'ils donnent . Là encore en faisant le tri, en modifiant certaines choses : ils «bricolent », «concoctent ». On observe une volonté de se démarquer des rôles sociaux et de l'image qu'ils ont de la génération précédente, mais en même temps, ils se définissent à partir de figures identificatoires de même sexe, ce qui favorise la pérennité des rôles sexuels. Comme l'a déjà remarqué Kaufmann (1994), les rôles se réinventent, mais chacun réinvente les mêmes rôles ; et redouble en le concrétisant, un sens qui est socialement et historiquement construit.

\section{Les mondes lexicaux des mères et des pères}

La méthodologie proposée par le logiciel Alceste permet de mettre en évidence, à travers l'analyse d'un corpus particulier - ici les 50 entretiens - les lieux usuels investis par les énonciateurs ; c'est la redondance des traces lexicales, des mots utilisés par les énonciateurs pour construire leur discours, qui permet de repérer les lieux les plus fréquentés. II s'agit de trouver la trace d’un « lieu de la pensée », d'un lieu de l'énonciation. Une analyse traitant séparément les discours des hommes et ceux des femmes a dégagé les mondes lexicaux des unes et des autres, et saisi ainsi la spécificité du positionnement de chacun des deux groupes. Cette analyse contribue à décrire le 
rapport que les personnes des deux catégories de sexe entretiennent avec leur expérience de parent ; rapport construit et verbalisé dans un contexte interlocutoire particulier tel que l'entretien en face en face.

\section{Traces lexicales représentatives des discours des hommes et des discours des femmes}

On a trouvé pertinent d'analyser les traces lexicales selon 7 dimensions (non pas définies a priori, mais à partir des deux listes dégagées par I 'analyse)

Lecture des cellules du tableau:

1. unités lexicales dont le Khi2 se situe entre 71 et 10

2. Unités lexicales dont le Khi2 se situe entre 10 et 6

3. Unités lexicales dont le Khi2, inférieur à 6 est encore significatif

\begin{tabular}{|c|c|c|}
\hline Dimensions & CLASSE 1 (Hommes) & CLASSE 2 (Femmes) \\
\hline Les autres et moi & $\begin{array}{l}\text { 1. Rapport, on, femme, époux, papa, } \\
\text { père, mamie, } \\
\text { 2. Paternel, échange, nous revenu } \\
\text { 3. Nos, grands-parents, répartition, } \\
\text { système, fonctionnement, équilibre, } \\
\text { dialogue, désaccord, relation, } \\
\text { association }\end{array}$ & $\begin{array}{ll}\text { 1. Je, me, moi, suis étais, lui, ils, mère, } & \text { maman, bébé, sœur } \\
\text { 2. Maternel, mari, leur, mon, elle, tu } \\
\text { 3. Grossesse, instinct }\end{array}$ \\
\hline Verbes & \begin{tabular}{|l} 
2. \\
Imposer \\
(serai), transformer secouer, solliciter, \\
imaginer, espérer, correspondre, \\
inscrire, descendre \\
3. Gérer, consacrer, décharger, projeter, \\
amener, raisonner, remarquer, estimer, \\
réclamer, obtenir
\end{tabular} & \begin{tabular}{|l} 
1. \\
Faire, trouver, être (suis, étais), être prête, \\
aller \\
Avoir (ai), laisser, créer, terminer, dire \\
(conjugué) appliquer, conseiller, laver, \\
ranger \\
3. Construire, élever, aider, appliquer, garder, \\
refaire, recommencer, manger, nettoyer, \\
réveiller, rentrer, chanter, dormir, gâter
\end{tabular} \\
\hline $\begin{array}{l}\text { Du côté de } \\
\text { l'éducation et des } \\
\text { valeurs }\end{array}$ & $\begin{array}{l}\text { 2. Humain, ordre, autorité, apprentissage } \\
\text { sévère, rigueur, devoir, } \\
\text { expérience, qualité } \\
\text { 3. Apprendre, éveiller, curieux, interdit, } \\
\text { pédagogie, exemple, souple, tolérance }\end{array}$ & 1. Honnête, indépendant \\
\hline Du côté des affects & $\begin{array}{l}\text { 1. Sévère, tempérament, intime, présent } \\
\text { 2. Humeur, affection, simple, normal, } \\
\text { difficile }\end{array}$ & $\begin{array}{l}\text { 1. Rire, bien, crier } \\
\text { 2. Génial, cool, plaisir, adorer, super, gentil, } \\
\text { culpabiliser, confiant } \\
\text { 3. Échec, inquiet, indépendant, content, déçu, } \\
\text { pire, chier, ennuyer, gêner, sacrifier }\end{array}$ \\
\hline La vie au jour le jour & 1. Concret, rapport, apprentissage, & 1. Rire, enceinte \\
\hline
\end{tabular}




\begin{tabular}{|c|c|c|}
\hline & $\begin{array}{l}\text { 2. Principal, fréquent } \\
\text { Pratique, expérience présent, baby- } \\
\text { sitting, limite, solliciter, secouer, } \\
\text { décision } \\
\text { 3. Contact, matériel, réalité, nourrir, } \\
\text { nourriture, fessée, gifle }\end{array}$ & $\begin{array}{l}\text { 2. laver, ranger, peinture, bordel, aide } \\
\text { 3. Manger, nettoyer, aide déjeuner, repas, } \\
\text { linge, réveiller, rentrer, danse, histoire, } \\
\text { piscine, chanter, dormir, bêtise, dent, } \\
\text { malade, bisou, câlin, gâter, peau, } \\
\text { poussette }\end{array}$ \\
\hline Espace et temps & $\begin{array}{l}\text { 1. Près } \\
\text { 2. Ici, contexte, crèche, spectacle, vis-à- } \\
\text { vis, hier, permanence, ponctuellement, } \\
\text { 3. Paris, banlieue, quartier, aujourd'hui, } \\
\text { auparavant, demain, avenir }\end{array}$ & $\begin{array}{l}\text { 1. Foyer, heure } \\
\text { 2. Maison, travail, journée, congés, déjà, } \\
\text { halte-garderie } \\
\text { 3. Domicile, garde, école, boulot, sur, chez, } \\
\text { continu, début, départ, nuit, soir, mi-temps, } \\
\text { tard, toujours, pendant }\end{array}$ \\
\hline Mots divers & $\begin{array}{l}\text { 1. Etc., aspect, maximum par, } \\
\text { généralement, aspect, par, ou } \\
\text { 2. Indéniable, dernier, nécessaire, } \\
\text { pleinement, cause, intellectuel, } \\
\text { fondamental, global, phase } \\
\text { 3. II me semble, tout à fait }\end{array}$ & $\begin{array}{l}\text { 1. Justement, ben, Anaïs, Antoine, } \\
\text { 2. Et, évidemment, Marc, Emma, Alain, } \\
\text { Manu, parce que, bordel, Stéphane, } \\
\text { Alphonse, grosso modo, Victor, Théodore, } \\
\text { oui } \\
\text { 3. De plus en plus, plein }\end{array}$ \\
\hline
\end{tabular}

Les mots associés aux discours des femmes évoquent l'univers de l'enfance et un rapport au temps concret, journalier; ils sont affectivement connotés. Les verbes d'action, concrets, abondent. Ils témoignent d'une expérience intime, individuelle et intersubjective, d'actions domestiques, relationnelles, inscrites dans le quotidien. Les traces lexicales caractérisant les discours féminins donnent l'idée d'une posture active, pratique, sensible, de l'ordre de la «métis ». Les mots associés aux discours masculins sont plutôt abstraits, les valeurs morales très présentes, les affects peu exprimés. Les hommes parlent de façon conceptuelle de l'éducation et de l'enfant, du fonctionnement du couple et des rapports familiaux. La dimension formelle, désincarnée, des traces lexicales des pères est évidente. Ce qui caractérise ces derniers, c'est leur propension à parler en termes abstraits de choses qui chez les femmes renvoient au contraire à des expériences que I 'on sent ancrées dans le réel, dans le vécu, dans la «chair » pourrait-on dire. On note chez les hommes, une posture plus distanciée, un regard plus formel, des traces lexicales illustrant une posture extérieure, retirée, réflexive. 


\section{Des mots pour des rôles}

Lorsque les parents ont eu à parler, face à un/e interviewer/e, de leur expérience parentale, (de l'organisation familiale à l'arrivée de l'enfant, des négociations avec le conjoint, de leur conception du rôle de parent), les femmes ont décrit cette expérience de l'intérieur, les hommes l'ont plutôt commentée de l'extérieur. Les interviewés ont produit un discours social. Leur parole doit être comprise ainsi : résultat de ce que chacun est en mesure de dire dans une situation sociale particulière (Bourdieu, 1994). Soulignons le fait que les entretiens ont été menés au domicile des personnes, ce qui n'est pas neutre, le foyer étant encore le lieu symbolique de la féminité (Kaufmann, 1988). Ajoutons aussi que dans la plupart des cas, ce sont les mères qui ont répondu à notre appel, et qui ont ensuite transmis notre requête à leur conjoint ; la place symbolique occupée par les uns et les autres n'est pas la même.

Dans la perspective de notre travail, «parler de », «parler sur » est une pratique sociale, illustrant un rapport - à la parentalité - qui se décline différemment pour les hommes et les femmes. Les mondes lexicaux des uns et des autres se distinguent. Symboliquement hommes et femmes n'occupent pas dans les mêmes lieux («topoï», Boltanski \& al. 1991) les unes se situant résolument du côté des activités et tâches d'éducation et d'organisation, les autres se situant plutôt du côté de la « réflexion sur ».

A l'instar de Singly (1990), on peut faire l'hypothèse d'une spécialisation homme-femme, et supposer que celle-ci permet au couple de résoudre une forme de contradiction : entre d'une part la logique d'efficacité - stratégique et instrumentale - et d'autre part la logique de distanciation et de subjectivation (Boutinet, 1998, Dubet, 1994).

\section{Quelle compréhension du partage?}

Ce qui est également intéressant à analyser, c'est le «rapport » que les individus entretiennent à leur expérience de la parentalité et à celle de leur conjoint. II semble y avoir un écart entre le principe d'égalité et d'équité, affirmé par l'ensemble des personnes, et la répartition réelle des tâches entre les hommes et les femmes (les femmes plus les hommes affirment que les tâches sont partagées : «on partage tout »). Pourtant ce partage n’est pas équitable, ni en termes de temps, ni en termes de nature des tâches. L'organisation domestique et la prise de rôles semblent 
Revue Connexions, n`82, 2004, pp.185-201

s'effectuer naturellement, sans discussion et ne semblent pas véritablement interroger (ou être interrogées par) les personnes. Les femmes de notre étude n'ont exprimé sur ce point, ni revendications, ni plaintes, ni ressentis négatifs. On peut s'interroger sur ce «mutisme » vis-à-vis d'une question souvent « brûlante » dans les débats sociaux.

Mères de très jeunes enfants, souvent jeunes elles-mêmes, pour la plupart installées depuis peu dans la conjugalité, bénéficiant de conditions d'existence favorables, elles semblent s'épanouir dans leur contexte de vie ; on sait que le sentiment amoureux, à la base du lien conjugal est peu compatible avec la revendication de parité entre les genres, et que c'est « à l'intérieur des rapports conjugaux et familiaux, sous couvert de l'amour, que continue à se reproduire, aujourd'hui comme hier, l'inégalité fondamentale entre homme et femme » (Bihr \& al., 2002, p.143).

Par ailleurs, il faut s'arrêter sur les notions de partage et de répartition. Remarquons en premier lieu qu'un partage n'est pas forcément équitable ; ainsi, si les femmes et les hommes estiment tous que les tâches sont partagées - et sans doute en effet le sont-elles - ils n'ont rien dit de l'équité de ce partage (les questions posées n'ont pas abordé directement la question de l'équité). Dans la notion de partage telle qu'elle est perçue chez nos sujets, il y a l'idée d'un partage des idées : au sein du couple il y a accord sur la manière dont les choses se passent, accord tacite qui ne fait pas l'objet de discussion. Kaufmann (1998) a bien montré que la tendance dominante est à se laisser porter par des ajustements implicites, plutôt que de négocier ouvertement ; et « le partage des tâches s'accomplit sous la pression de l'inégale disponibilité des conjoints selon les différents moments de la journée ou de la semaine »(Bihr \& al., 2002, p136).

Un extrait d'entretien illustre la manière dont se répartissent les tâches au sein d'un couple. II s'agit d'une femme de 33 ans, mère de deux enfants de 6 et 2 ans. Responsable du service fiscal d'une entreprise, elle travaille à mi-temps, quatre jours par semaine. Son conjoint est directeur d'une entreprise d'expertise comptable (couple catégorisé dans la classe 3). Cet extrait permet de comprendre l'aspect naturel du processus de prise de rôles (naturel et spontanéité que l'ensemble des parents de notre étude revendiquent)

«- Le partage des tâches... La cuisine, c'est plutôt moi ; le ménage, c'est quand même plutôt moi et puis la jeune fille qui vient aussi les garder vient faire du ménage. Et puis mon mari, lui, s'occupe pas mal de tout ce qui est... Plutôt lui, c'est l'amusement des enfants, c'est-à-dire que 
Revue Connexions, $\mathrm{n}^{\circ} 82$, 2004, pp.185-201

moi, je sais pas m'amuser avec les enfants, lui sait bien le faire, donc c'est lui qui fait ça et puis évidemment, tout ce qui est bricolage dans la maison. Ca, c'est quelque chose de très classique. C'est vraiment... (rires)

- Vous avez des rôles différenciés ?

- Disons que, attendez... S'il faut faire une vaisselle, il la fait, sans jamais demander, il la fait aussi naturellement. Je veux dire de façon générale puisqu'on est dans la généralité, c'est plutôt moi qui fais à manger, c'est plutôt moi qui m'occupe de tout ce qui est linge, etc., c'est plutôt moi, tout ça.

- Est-ce qu'il y a une différence entre l'organisation de la semaine et du week-end?

- Oui, c'est à dire que le week-end mon mari m'aide beaucoup plus que pendant la semaine parce qu'il a un boulot monstre pendant la semaine, ce qui fait que le week-end, c'est plutôt... Là, il y a un vrai partage des tâches pendant le week-end. Pendant la semaine, c'est plutôt moi.

- Cette organisation de la vie quotidienne s'est mise en place de façon naturelle?

- Ha oui, j'ai de la chance, il met la main à la pâte tout à fait spontanément. Vraiment. »

II est intéressant de noter la manière dont la personne en vient à préciser, progressivement, comment s'opère le partage des tâches : visiblement ce partage n'est pas équitable. Mais, comme le dit Kaufmann, (1988, p.112), « ce qui compte dans l'ajustement ce n'est pas que ce qui en résulte puisse être considéré comme juste par un observateur extérieur, c'est que les deux conjoints soient d'accord dans leur définition de la hiérarchie des valeurs, y compris si la division des tâches est très inégalitaire ».

Nous avons en effet noté que les couples interviewés présentent une grande homogénéité sur le plan des valeurs et des orientations à donner à leur vie et à l'éducation de leur enfant. Se dégage des entretiens une impression d'harmonie, d'accord «silencieux » sans doute antérieur à la naissance de l'enfant, en cohérence avec l'aspect spontané, souple, de la dynamique conjugale. L'ethos que le couple a construit dans les premières années de vie commune lui permet d'agir au quotidien par ajustements successifs, sans que soit nécessaire une discussion sur les orientations et les principes (une femme dit à propos de son conjoint devenu père : « je m'en doutais un peu, qu'il serait comme ça »). On peut alors mieux comprendre ce qui rend les ajustements si solides ; en effet, c'est lorsque « la négociation ne se vit pas comme telle qu'elle est la plus efficace » (Kaufmann, 1988, p. 112). Les parents semblent attachés à cette conception de la parentalité en tant que pratique spontanée : ils considèrent dans l'ensemble, qu'être parent ne s'apprend pas, ou s'apprend sur le tas, qu'il s'agit d'agir au « feeling », à l'intuition, au jour le jour ; certains parlent de 
Revue Connexions, $n^{\circ} 82,2004$, pp.185-201

«bricolage », beaucoup ont exprimé leur bonheur à être parent. L'exercice de la parentalité n'est pas dominé par la rationalité (ce qui contraste avec la sphère professionnelle).

A ce niveau de l'étude, on pourrait formuler l'hypothèse que l'attachement des parents à la dimension spontanée - et donc peu réflexive - de la parentalité conduit à inhiber sans doute les dispositions à y porter un regard critique et réflexif (Ricard-Fersing, 2001, Lahire, 2002). Les analyses de Boltanski et Thévenot (1991) contribuent également à éclairer nos résultats : dans l'espace social coexistent divers mondes - des « cités »- , auxquels sont attachés des systèmes de valeurs différents et entre lesquels évoluent les acteurs sociaux. Dans ces «modèles régionaux d'action ", la part de conscience et de réflexivité n'est pas la même et les acteurs sociaux raisonnent et agissent différemment selon les lieux qu'ils occupent. Ainsi, certaines actions sont de l'ordre de la justice, ont la réciprocité pour règle, prennent appui sur des principes d'équivalence ; d'autres au contraire relèvent de l'amour («Agapè ») et se manifestent par le don, la gratuité, le renoncement au calcul et à la justice. Ainsi, il apparaît que les femmes de notre étude ont pensé et parlé leur expérience «selon la loi d'Agapè », n'adoptant pas un point de vue général, distancié, qui les eût fait « basculer hors de l'amour », hors de l'altruisme et du dévouement (Boltanski, 1990, Molinier, 2003).

\section{Conclusion}

Être parent d'un jeune enfant, c'est notamment s'impliquer fortement dans l'organisation et la logistique du quotidien, et les mères, plus que les pères, prennent cela en charge. Les deux types d'analyses (qualitative et quantitative) permettent de le constater. Si l'égalité homme-femme est au moins en principe - aujourd'hui acceptée, il n'en reste pas moins que la problématique identitaire et l'expérience parentale s'expriment de manière spécifique pour les uns et les autres. L'identité des hommes et des femmes s'élabore à partir de figures identitaires spécifiques et se fonde sur une division des rôles et des tâches.

Les femmes que nous avons rencontrées, qui ont gardé une insertion sur le plan professionnel, se présentent comme préoccupées par la régulation de leurs deux champs d'activités : l'activité professionnelle et la vie familiale. Ceci vient nuancer le modèle traditionnel de la féminité/masculinité (les femmes « du côté » de l'affectivité, l'émotion, la dynamique relationnelle, 
Revue Connexions, $\mathrm{n}^{\circ} 82$, 2004, pp.185-201

les hommes « du côté » de l'action). En fait, les femmes, par leur investissement dans les tâches quotidiennes, leur engagement dans le «gouvernement domestique » (Fraisse, 2000) sont résolument tournées vers l'action, la décision, la planification. Les hommes apparaissent plus extérieurs... à la fois en termes d'implication quotidienne et sur le plan de la pensée, leur discours exprimant une certaine « distance». Au cours des entretiens, ils ont semblé impliqués au niveau de la « réflexion sur », plutôt qu'au niveau de « l'action pour ».

On constate avec Lahire (2002, p. 420) que les croyances et les idéaux des hommes et des femmes «peuvent être clairement du côté du partage égalitaire des tâches alors même que des habitudes, des propensions à agir contraires peuvent être mises en œuvre ». En fait, le vrai défi des femmes paraît se situer moins du côté d'un partage équitable des tâches avec leur conjoint, que dans la réalisation d'un « double » objectif : être mère et mener une activité professionnelle. Cela suppose deux expériences de socialisation différentes, s'accompagnant, sur le plan psychique, de la coexistence de dispositions variées, et sans doute parfois contradictoires. Pour prolonger cette étude, il serait judicieux d'affiner la compréhension des dissonances psychiques, d'étudier la manière dont les personnes parviennent à négocier, sur le plan intrapsychique, entre différentes dispositions à agir et à penser. L'approche sociopsychologique nous semble tout à fait pertinente pour avancer dans cette voie.

\section{Bibliographie}

Berger, P. \& Kellner, H., 1988, Le mariage et la construction de la réalité, Dialogue, $\mathrm{n}^{\circ} 102$, pp.6-21.

Berger, P. \& Luckmann, T., 1966, La construction sociale de la réalité, Paris, Méridiens Klincksieck, 1986 pour la traduction française.

Bergonnier-Dupuy, G., 2000, Les processus éducatifs intra-familiaux, Les Sciences de l'Éducation pour l'Ère Nouvelle, 33, 4.

Bergonnier-Dupuy, G., Bouissou, C., 2003, Identité parentale et sentiment de compétence, Rapport final de recherche et note de synthèse, Paris, CNAF.

Bihr, A \& Pfefferkorn, 2002, Hommes et femmes quelle égalité ? Paris, Les éditions de l'atelier, Editions ouvrières. 
Boltanski, L., 1990, L’amour et la justice comme compétences, Paris, Métailié.

Boltanski, L. \& Thévenot, L., 1991, De la justification, Paris, Gallimard.

Bouissou, C. \& Bergonnier-Dupuy, G., 2004, Être père, être mère: deux expériences singulières, Sciences de l'homme et sociétés/Cultures en mouvement, pp20-22, $n^{\circ} 69$.

Bourdieu, P., 1994, L'illusion biographique, in Raisons pratiques, sur la théorie de l'action, Paris, Seuil, chapitre 3, annexe 1.

Boutinet, JP., 1998, L'immaturité de la vie adulte, Paris, Puf.

Bulard, M., 2003, Sexisme ordinaire au travail, Manière de voir, n68, pp. 36-41.

Cicchelli, V., 2001, La construction du rôle maternel à l'arrivée du premier enfant, Recherches et Prévisions, n63, pp.33-45.

Corcuff, P. , 2003, Bourdieu autrement. Fragilités d'un sociologue de combat, Paris, Textuel .

Dejours, C., 1988, Le masculin entre sexualité et société, Adolescence, vol 6, n¹, pp 89-116

Dubet, F., 2000, Rôle et expérience, in L'analyse de la singularité de l'action, Paris, Puf, pp.7183.

Dubet, F., 1994, Sociologie de l'expérience, Paris, Seuil.

Elias, N., 1973, La civilisation de mœurs, Paris, Calmann-Lévy.

Fraisse, G., 2000, Les deux gouvernements : la famille et la cité, Paris, Folio.

Giddens, A., 1987, La constitution de la société, Paris, Puf.

Junter-Loiseau, A., 1997, La notion de conciliation professionnelle et familiale, Cahiers du Genre, n²4, pp 73-98.

Kaufmann, JC., 1998, Il faut se méfier du balai, Revue Sciences Humaines, n88.

Kaufmann, JC, 1994, Rôles et identité : l'exemple de l'entré en couple, Cahiers Internationaux de Sociologie, Vol. XCVII, pp. 301-328.

Kaufmann, JC, 1991, Les routines conjugales, Sciences Humaines n 9, pp.43-45.

Kaufmann, JC., 1988, La chaleur du foyer. Analyse du repli domestique, Paris, Méridiens Klincksieck.

Lahire, B., 2002, Portraits sociologiques. Dispositions et variations individuelles, Paris, Nathan. 
Revue Connexions, $n^{\circ} 82,2004$, pp.185-201

Lahire, B., 2001, De la théorie de l'habitus à une sociologie psychologique, in Le travail sociologique de Pierre Bourdieu, Dettes et critiques Paris, Nathan.

Mead, GH., 1933, L'esprit, le soi et la société, Paris, PUF (1963 pour la traduction).

Meyerson, I., 1948, Les fonctions psychologiques et les œuvres, Paris, Vrin.

Molinier, P., 2003, L'énigme de la femme active, égoïsme, sexe et compassion, Paris, Payot.

Molinier, P., 2002, Féminité sociale et construction de I'identité sexuelle : perspectives théoriques et cliniques en psychodynamique du travail, L'Orientation Scolaire et Professionnelle, $31, n^{\circ} 4$, pp. 565-580.

Reinert, M., 1997, Les mondes lexicaux des six numéros de la revue «le surréalisme au service de la Révolution ", Cahiers du centre de recherche sur le surréalisme, $n^{\circ} X V I, p p$. 270-302.

Ricard-Fersing, E., 2001, Vers une philosophie de la pratique réflexive, Carrefours de l'éducation, n¹2, pp. 67-81.

Ricoeur, P., 1990, Soi-même est un autre, Paris, Seuil.

Schön, D., 1994, Le praticien réflexif. A la recherche du savoir caché dans l'agir professionnel, Les éditions logiques.

Singly de, F., 1990, L’homme dual, le Débat, n61, pp138-151

Tap, P., 1988, La société Pygmalion ? Intégration sociale et réalisation de la personne. Paris, Dunod.

Wallon, H. ,1946, Le rôle de l'autre dans la conscience du moi. Enfance, nº spécial (1959) 3-4, pp.279-286. 